\title{
A "bridge" technique to replace the obstructed single-J stent in the patient with ileal conduit urinary diversion: a case report
}

\author{
Kunlin Yang ${ }^{1,2,3}$, Xuesong $\mathrm{Li}^{1,2,3}$, Gang Wang ${ }^{1,2,3}$ \\ ${ }^{1}$ Department of Urology, Peking University First Hospital, Beijing, China; ${ }^{2}$ Institute of Urology, Peking University, Beijing, China; ${ }^{3}$ National \\ Urological Cancer Center, Beijing, China \\ Correspondence to: Gang Wang. Department of Urology, Peking University First Hospital, Beijing, China; Institute of Urology, Peking University, \\ Beijing, China; National Urological Cancer Center, No. 8 Xishiku St, Xicheng District, Beijing 100034, China. Email: drwanggang@163.com.
}

\begin{abstract}
The ureteroileal anastomotic stricture is a complication of ileal conduit urinary diversion. To prevent the hydronephrosis and protect the renal function, a single-J ureteral stent may be needed. However, the most common complication of these patients is single-J stent obstruction. To solve this problem, we describe an easy, useful and low-cost technique to replace the obstructed ureteral stent under radiographic guidance without intervention by flexible cystoscopy or percutaneous nephrostomy. The key steps of our procedure are to identify the location of the stricture, to place the super smooth guide wire into pinhole of the obstructed single-J stent and to get the super smooth guide wire and 5-Fr ureteral catheter across the stricture. Our case was a 40-year-old male patient who was diagnosed as pelvic lipomatosis and received ileal conduit urinary diversion 3 years ago. The left-side ureteroileal anastomotic stricture occurred 1 year after surgery. He refused to repair the stricture by open or other minimal invasive surgery. He regularly changed his ureteral stent with intervals of three months. As the stent was obstructed by the stone, the guide wire couldn't be inserted through the primary ureteral stent. We used our "bridge" technique to solve his problem successfully. No bleeding and no urinary tract infection were observed after intervention. The urine from the ureteral stent was fluent. We think that this "bridge" technique may be a good choice for the replacement of the obstructed single-J stent in the patients of ileal conduit urinary diversion.
\end{abstract}

Keywords: Ureteral stent; ureteral obstruction; ileal conduit; urinary diversion; case report

Submitted Aug 18, 2020. Accepted for publication Nov 04, 2020.

doi: $10.21037 /$ tau-20-1186

View this article at: http://dx.doi.org/10.21037/tau-20-1186

\section{Introduction}

The ureteroileal anastomotic stricture is a bothering complication of ileal conduit urinary diversion. It has been reported that the ureteroenteric stricture rate ranged from $2.6 \%$ to $14.5 \%$ after radical cystectomy $(1,2)$, which might cause hydronephrosis and loss of renal function. The ureteral stent or percutaneous nephrostomy are often temporary treatments to maintain proper renal function. For most of the patients, they need to undergo balloon dilation or other surgeries of repair $(3,4)$. In the patients with ureteral stent, the most common complication is the ureteral stent obstruction, which may require retrograde or anterograde endoscopic procedures to reinsert a new stent
$(5,6)$. To facilitate the management of this condition, we report an easy and useful technique to change an obstructed ureteral stent by two doctors without any other equipment. We present the following case in accordance with the CARE reporting checklist (available at http://dx.doi.org/10.21037/ tau-20-1186).

\section{Case presentation}

The patient was a 40 -year-old man, who received ileal conduit urinary diversion because of pelvic lipomatosis 3 years ago. The left-side ureteroileal anastomotic stricture occurred 1 year after surgery. He refused to repair the stricture by open or other minimal invasive surgery. So, he 

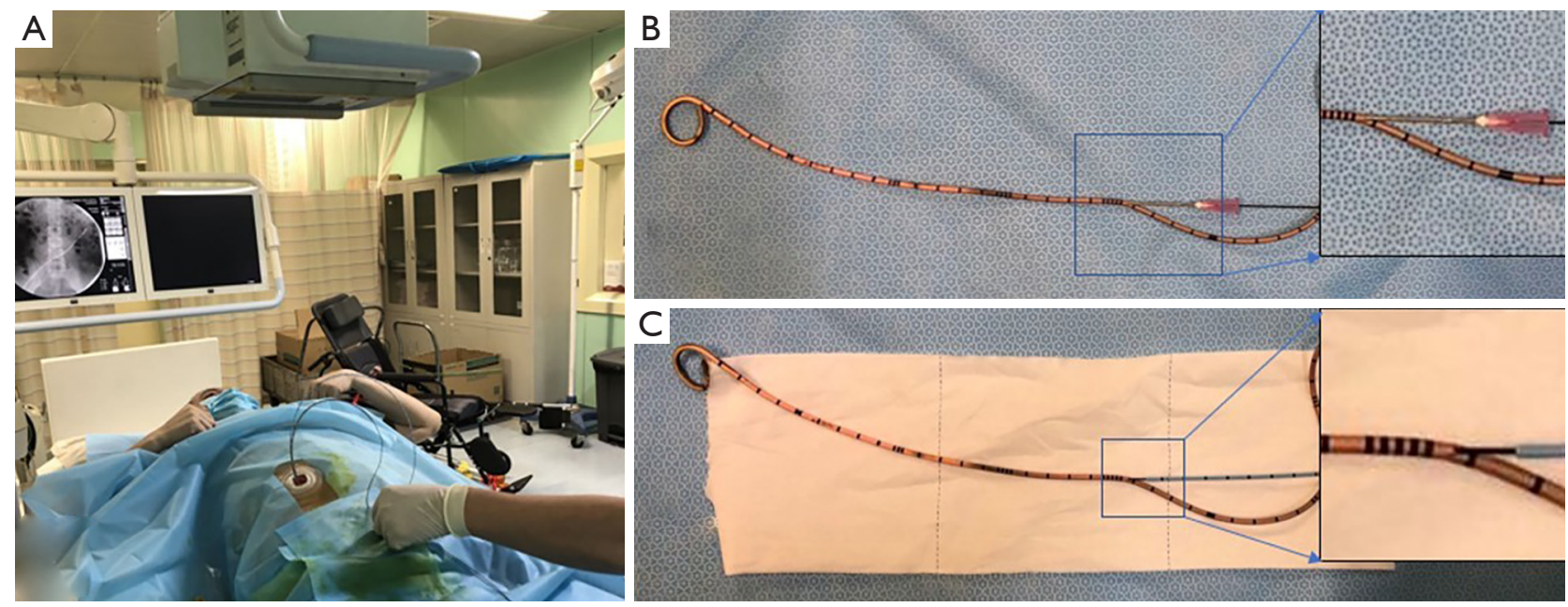

Figure 1 The position of the patient and some tips about the procedure. (A) Supine position of the patient; (B) a 20-mL syringe needle is inserted into the obstructed ureteral stent and a super smooth guide wire is placed into the stent about $1 \mathrm{~cm}$ through the pinhole; (C) a stiff and open 5-Fr ureteral catheter is inserted through the super smooth guide wire.

regularly changed his ureteral stent with intervals of three months. As the stent was obstructed by the stone, the guide wire couldn't be inserted through the primary ureteral stent after trying many times. We decided to use our "bridge" technique to change his obstructed ureteral stent.

The patient was placed supine under X-ray machine (shown in Figure 1). As the ileal conduit of this patient was Wallace procedure, we had known the possible position of the stricture which was a very important mark point. We could call it point A (shown in Figure 2A). After we identified the general position of the stenosis, the single-J stent was pulled out until the point $A$ on the stent just beyond the ileal stoma (shown in Figure 2B). We needed to perform these procedures carefully under radiographic guidance to always keep the single-J stent in the ureter. A 20 - $\mathrm{mL}$ syringe needle was inserted into the ureteral stent on the proximal position of the marked point A (shown in Figure 1B). A super smooth guide wire was placed into the ureteral stent about $1 \mathrm{~cm}$ through the pinhole (shown in Figure $1 B$ and Figure 2B). Then, the needle was pull out from the super smooth guide wire. A stiff and open $5-\mathrm{Fr}$ ureteral catheter was inserted through the super smooth guide wire (shown in Figure 1C and Figure 2B). The single-J stent was pushed in by an ordinary guide wire until the super smooth guide wire and the 5-Fr ureteral catheter passed the ureteroileal anastomotic stricture which could be identified under radiography (shown in Figure 2C). We pulled out the super smooth guide wire $2 \mathrm{~cm}$ when fixing the 5-Fr ureteral catheter to detach the super smooth guide wire from the obstructed ureteral stent. Then, we inserted the super smooth guide wire and the 5-Fr ureteral catheter until they reached the renal pelvis (shown in Figure 2D). We removed the obstructed ureteral stent (shown in Figure 2E). A new single-J stent was placed in proper position through the super smooth guide wire (shown in Figure $2 F$ and Figure $2 G$ ). We found the removed ureteral stent was obstructed by stone (shown in Figure $2 H$ ).

After intervention, no bleeding and no urinary tract infection were observed. The urine from the ureteral stent was fluent. The ultrasound showed no hydronephrosis on two side of the kidneys.

All procedures performed in studies involving human participants were in accordance with the ethical standards of the institutional and/or national research committee(s) and with the Helsinki Declaration (as revised in 2013). Written informed consent was obtained from the patient.

\section{Discussion}

The critical point of our procedure is to use the obstructed ureteral stent like a "bridge" to take the new guide wire across the stenosis. So, we named it as "bridge" technique. By using this "bridge" technique, we have successfully changed the obstructed single-J stents two times for this patient. Before we started this method, we preferred to perform retrograde flexible cystoscopy or anterograde 

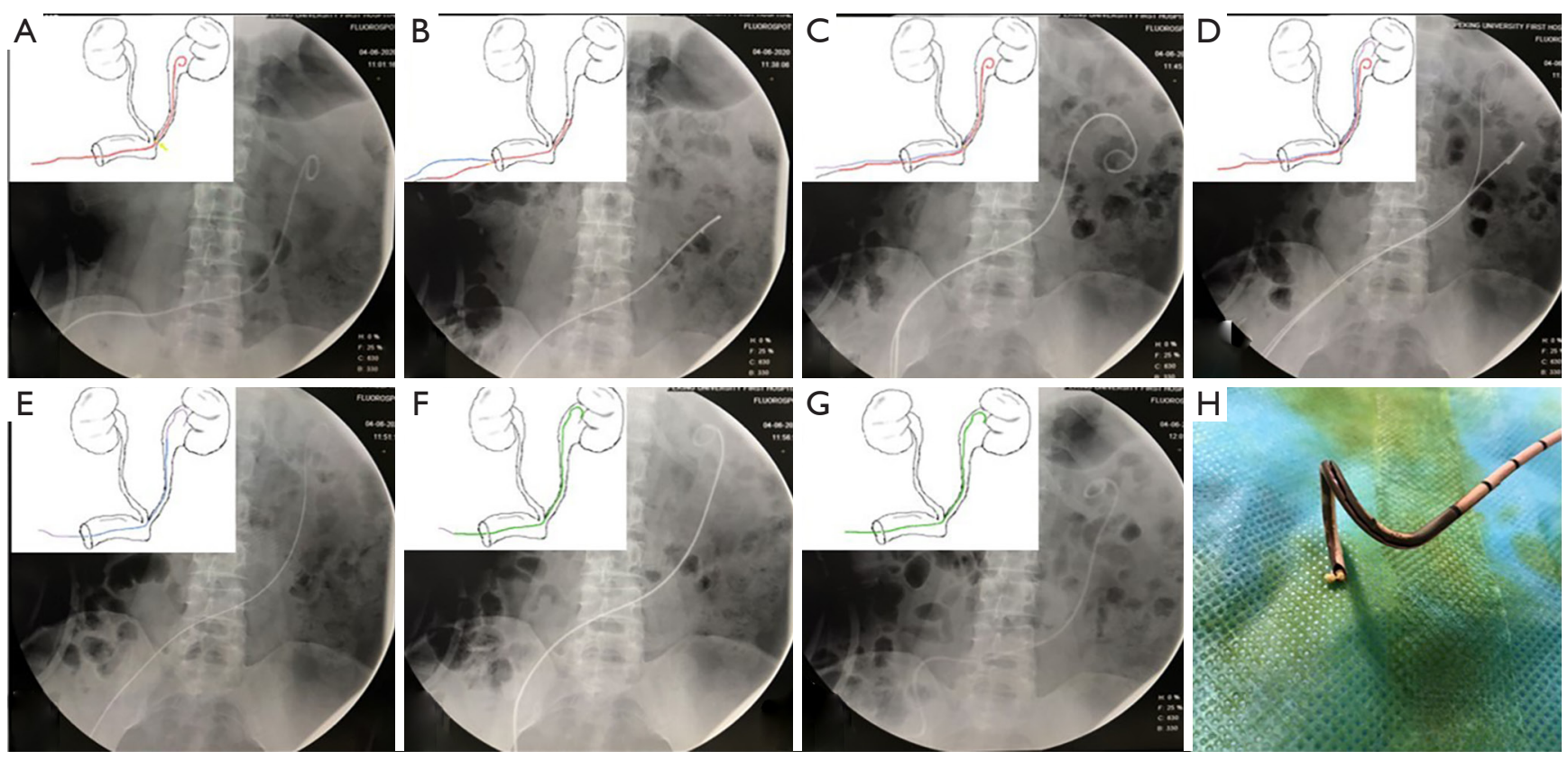

$\mathrm{F}$
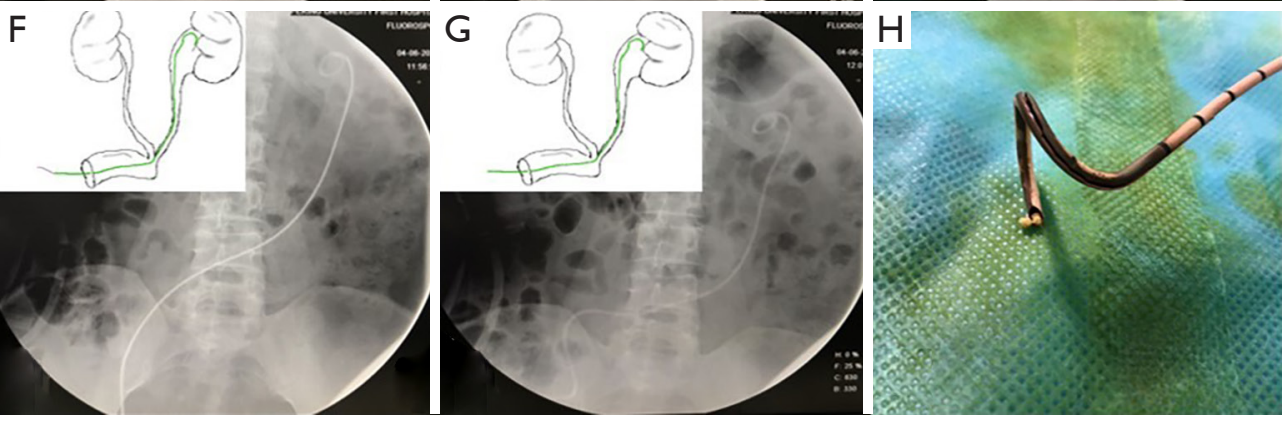

Figure 2 The main steps of the procedure under the radiographic guidance. (A) Identification of the stricture shown by yellow arrow; (B) the obstructed stent is pulled out. The super smooth guide wire and 5-Fr ureteral catheter are attached with the obstructed stent from a pinhole; (C) the obstructed stent is pushed back by an ordinary guide wire to get the super smooth guide wire and $5 \mathrm{Fr}$ ureteral catheter across the stenosis; (D) the super smooth guide wire is detached from the obstructed stent and reinserted into the renal pelvis; (E,F,G) a new ureteral stent is inserted into the renal pelvis through the super smooth guide wire; $(\mathrm{H})$ the stone causing the obstruction.

percutaneous nephrostomy to place a new single-J stent for the patients.

In 2000, Saito et al. reported a technique to change the obstructed ureteral stent in ileal conduit (7). But we found it was very difficult to insert 5 -Fr ureteral catheter through the guide wire and detach the guide wire from the obstructed ureteral stent by their method.

Our "bridge" technique has three important tips:

Firstly, we must identify the possible location of the ureteroileal anastomotic stricture. If the position of the stenosis couldn't be confirmed, we can inject the contrast agent into the ureter and the ileal conduit from the obstructed ureteral stent under radiography.

Secondly, we suggest that the guide wire placed into pinhole should be a super smooth one. It will make it easy when we reinsert the guide wire into the renal pelvis.

Thirdly, the open 5-Fr ureteral catheter should be relatively stiff. If it was too soft, we can't detach the guide wire from the obstructed single-J stent. We must ensure the $5 \mathrm{Fr}$ ureteral catheter and the super smooth guide wire are reinserted into ureter crossing the stenosis simultaneously when we push the obstructed ureteral stent.

Our "bridge" technique also has its limitations. We must perform this technique under radiography. If the ureteroileal anastomotic stricture was too narrow, maybe it will become more difficult. For this patient, he refused to repair the stricture. Actually, only the ureteral reconstruction can repair this kind of stricture completely.

We think our procedure can solve the problem similar with our case caused by the obstructed stent. It avoids further intervention by flexible cystoscopy or percutaneous nephrostomy and is low cost and time-saving. Another suggestion is that the single-J stent should be changed regularly. In conclusion, we report an easy and useful technique to replace the obstructed ureteral stent in patients with ileal conduit urinary diversion.

\section{Patient perspective}

"Thank you for my doctors to help me change this obstructed ureteral stent by using this method. I was scared to receive nephrostomy." 


\section{Acknowledgments}

The authors thank Yanpeng Xu for his help in radiographic guidance.

Funding: None.

\section{Footnote}

Reporting Checklist: The authors have completed the CARE reporting checklist. Available at http://dx.doi.org/10.21037/ tau-20-1186

Conflicts of Interest: All authors have completed the ICMJE uniform disclosure form (available at http://dx.doi. org/10.21037/tau-20-1186). The authors have no conflicts of interest to declare.

Ethical Statement: The authors are accountable for all aspects of the work in ensuring that questions related to the accuracy or integrity of any part of the work are appropriately investigated and resolved. All procedures performed in studies involving human participants were in accordance with the ethical standards of the institutional and/or national research committee(s) and with the Helsinki Declaration (as revised in 2013). Written informed consent was obtained from the patient.

Open Access Statement: This is an Open Access article distributed in accordance with the Creative Commons Attribution-NonCommercial-NoDerivs 4.0 International License (CC BY-NC-ND 4.0), which permits the non- commercial replication and distribution of the article with the strict proviso that no changes or edits are made and the original work is properly cited (including links to both the formal publication through the relevant DOI and the license). See: https://creativecommons.org/licenses/by-nc-nd/4.0/.

\section{References}

1. Goh AC, Belarmino A, Patel NA, et al. A Populationbased Study of Ureteroenteric Strictures After Open and Robot-assisted Radical Cystectomy. Urology 2020;135:57-65.

2. Evangelidis A, Lee EK, Karellas ME, et al. Evaluation of ureterointestinal anastomosis: Wallace vs Bricker. J Urol 2006;175:1755-8; discussion 1758.

3. Nassar OA, Alsafa ME. Experience with ureteroenteric strictures after radical cystectomy and diversion: open surgical revision. Urology 2011;78:459-65.

4. Schöndorf D, Meierhans-Ruf S, Kiss B, et al. Ureteroileal strictures after urinary diversion with an ileal segment-is there a place for endourological treatment at all? J Urol 2013;190:585-90.

5. Woods SD, Reisner GS. Ureteral stenting via an ileal conduit using a gastroscope. J Urol 2002;168:185.

6. Zaleski GX, Funaki B, Newmark G. Placement of retrograde nephroureteral stents through ileal conduits. AJR Am J Roentgenol 1998;170:1275-8.

7. Saito M, Sakaridani N, Yamamoto Y, et al. A simple technique to change an obstructed ureteral stent in patients with an ileal conduit. J Urol 2000;164:1604-5.
Cite this article as: Yang K, Li X, Wang G. A "bridge" technique to replace the obstructed single-J stent in the patient with ileal conduit urinary diversion: a case report. Transl Androl Urol 2021;10(1):532-535. doi: 10.21037/tau-20-1186 